\section{Liver infarction in a patient with Clostridium Difficile colitis. A possible connection?}

\section{To the Editor,}

Patients with acute leukemia are prone to develop infections with various microorganisms, including Clostridium difficile (CD). The evolution and complications of colitis with CD in these patients are far from being fully known and understood.

A 66-year-old female patient was diagnosed with an acute B-lymphoblastic leukemia. A central venous catheter placed in the left femoral vein was complicated with venous thrombosis. Anticoagulant treatment was given until the onset of medullary aplasia. The chemotherapy consisted of the HYPER-C VAD/ MTX/ARA-C protocol (cyclophosphamide, vincristine, doxorubicin and dexamethasone followed by methotrexate and cytarabine). After the first cycle of chemotherapy, 35-65\% blast cells remained in the bone marrow. One week after the second chemotherapy cycle, the patient developed a CD colitis, which was treated with vancomycin, orally. The patient had no blast cells in the peripheral blood when the bone marrow started to recover from the post-chemotherapy aplasia and started the prophylactic treatment with dalteparin 5000UI/ day, s.c. when the number of platelets increased over 30,000/ $\mathrm{mm}^{3}$. She suddently presented increased aminotransferase levels (up to $7217 \mathrm{U} / \mathrm{L}$ ) and total serum bilirubin level (up to $2.95 \mathrm{mg} / \mathrm{dl}$ ). Acute viral hepatitis A, B and E were excluded. Blood fibrinogen, fibrin monomers and D-dimers levels were increased. An angio-computed tomography (CT) detected an obstruction of the right branch of the portal vein and a large hypofixant area into the right liver lobe (Fig. 1), suggestive of liver infarction. Evolution of the patient was unfavorable: she developed severe liver failure followed by death.

Conventional clotting tests often offer abnormal results in patients with acute lymphoblastic leukemia, suggesting a bleeding tendency when, in fact, thrombosis is a higher risk [1]. Global clotting tests provide the results of the effect and interaction of multiple pathways and are superior to conventional clotting tests for the exploration of coagulation:



Fig. 1. CT with contrast agent: the right branch of the portal vein is permeable only adjacent to the trunk of the portal vein; large hypofixant area in the right lobe of the liver; ascended right hemidiafragm.

thromboelastography investigates whole-blood clot formation and fibrinolysis, and the study of thrombin generation explores the net balance between pro- and anticoagulant proteins in plasma [1]. Unfortunately, these global clotting tests, as well as those for exploring thrombophilia, are not routinely available. Our patient had no previous thrombotic events.

The age $>65$ years, prolonged hospitalization, neutropenia and immunosuppression, the exposure to methylprednisolone and broad-spectrum antibiotics for an enterococcal urinary infection were risk factors for CD colitis in our patient. Methotrexate does not commonly cause clotting disorders. Methotrexate and cytarabine might have liver toxicity (increased aminotransferase levels). Cytarabine can produce, rarely, intestinal necrosis and perforation, liver abscesses and Budd Chiari syndrome [2]. Intensive chemotherapy contributes to gastrointestinal mucosal inflammation and ulcerations. In addition, $\mathrm{CD}$ contributes to the disruption of the intestinal epithelial barrier, favoring extraintestinal infections with CD. 
A recurrent case of bacteremia and liver abscess produced by $\mathrm{CD}$ was published [2]. A possible bacterial translocation due to $\mathrm{CD}$ overgrowth in the intestine might explain the occurrence of the abscess. The spore-forming ability of CD could have allowed it to survive during antimicrobial therapy [2].

It has been observed that $\mathrm{CD}$ infection increases the risk of venous thromboembolism in patients from intensive care unit [3]. Clostridium difficile could pass through the damaged intestinal membrane and reach the portal circulation. The bacteria could activate neutrophils and release neutrophil extracellular traps, involved in thrombin generation through a platelet-dependent mechanism [4]. Bacterial toxins contribute to the growth of thrombin generation through the Rac1 signaling mechanism [5]. In addition, thrombin is involved in the activation of the inflammatory process, which results in the increased generation of thrombin.

Immunosuppressed patients, as was the case of our patient, are predisposed to develop CD colitis. Induction chemotherapy for acute leukemia increases the permeability of the intestinal wall to toxins and bacteria, allowing passage of $\mathrm{CD}$ into the portal vein and the liver. The inflammation caused by $C D$ could favor portal vein thrombosis even in the presence of thrombocytopenia.

\section{Romeo-Gabriel Mihaila ${ }^{1}$, Mariana Sandu ${ }^{2}$}

1) Faculty of Medicine, Lucian Blaga University of Sibiu, Hematology Department, Emergency County Clinical Hospital Sibiu; 2) Medical Imaging Department, Emergency County Clinical Hospital Sibiu, Romania

Correspondence: Romeo-Gabriel Mihăilă, romeomihaila@yahoo.com

Conflicts of interest: None.

DOI: $10.15403 /$ jgld-187

\section{REFERENCES}

1. Burley K, Salem J, Phillips T, et al. Evaluation of coagulopathy before and during induction chemotherapy for acute lymphoblastic leukaemia, including assessment of global clotting tests. Blood Cancer J 2017;7:e574. doi:10.1038/bcj.2017.54

2. Morioka H, Iguchi M, Kuzuya T, Mikamo H, Yagi T. Recurrent bacteremia and liver abscess caused by Clostridium difficile. Medicine (Baltimore) 2017;96:e7969. doi:10.1097/MD.0000000000007969

3. Barmparas G, Fierro N, Lamb AW, et al. Clostridium difficile increases the risk for venous thromboembolism. Am J Surg 2014;208:703-709. doi:10.1016/j.amjsurg.2014.05.025

4. Gould TJ, Vu TT, Swystun LL, et al. Neutrophil extracellular traps promote thrombin generation through platelet-dependent and plateletindependent mechanisms. Arterioscler Thromb Vasc Biol 2014;34:19771984. doi:10.1161/ATVBAHA.114.304114

5. Wang Y, Hwaiz R, Luo L, Braun OÖ, Norström E, Thorlacius H. Rac1 regulates bacterial toxin-induced thrombin generation. Inflamm Res 2016;65:405-413. doi:10.1007/s00011-016-0924-3

\section{Lipidomic signatures of colonic inflammatory bowel diseases: a pilot study}

\section{To the Editor,}

Inflammatory bowel diseases (IBD) are challenging conditions to diagnose and treat despite the fact that they have been known for over 50 years. They use important medical resources and may have a resounding psychosocial impact. Recently, alterations in lipid profiles have been incriminated in the pathophysiology of IBD, with phosphatidylcholine, sphingolipids and ceramides being involved in important processes such as apoptosis, proliferation, stress response, necrosis, inflammation and autophagy [1].

We designed an experimental study to realize a lipidomic fingerprint of colonic IBD and to identify lipid molecules that could serve as putative diagnostic biomarkers. Blood samples were collected from consecutive patients with either active left sided or extensive ulcerative colitis or active colonic Crohn's disease who had been admitted to a tertiary referral hospital. The diagnosis was established using clinical, serological, endoscopic and histological data. Patients with proctitis or

Table I. Demographic and clinical data of the subjects

\begin{tabular}{lccc}
\hline & Ulcerative colitis & Crohn's disease & Healthy controls \\
\hline Number of patients & 17 & 5 & 24 \\
Gender (female:male) & $6: 11$ & $3: 2$ & $14: 10$ \\
Average age (years) (range) & $39(18-65)$ & $43(38-59)$ & $29(24-40)$ \\
Disease localization and extension (number of & Left sided colitis: 7 & Colitis: 5 & Perianal lesions: 2 \\
patients) & Extensive colitis: 10 & CDAI: 182 \\
Average clinical severity & UCDAI: 7.6 & SES-CD: 12 \\
Average endoscopic severity & Mayo score: 2.3 & \\
Current medication for IBD (number of patients) & & 1 \\
Mesalazine & 9 & 2 \\
Budesonide & 0 & 1 \\
Azathioprine & 1 & 1 \\
Infliximab & 0 & 3 & \\
Adalimumab & 3 & \\
\hline CDAI: Crohn's Disease Activity Index; SES-CD: Simple Endoscopic Score for Crohn' Disease; UCDAI: Ulcerative \\
Colitis Disease Activity Index.
\end{tabular}




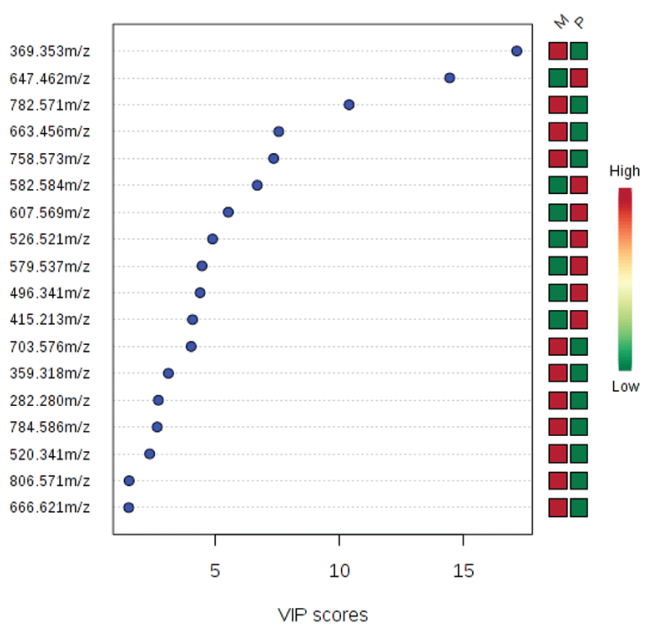

Fig. 1. VIP scores that discriminate between IBD patients $(\mathrm{P})$ and healthy subjects $(\mathrm{M})$. The colored scale indicates the variations between the two groups.

indeterminate colitis were excluded from the study, as well as patients $<18$ or $>70$ years old, those having a history of neoplasia, infectious diseases, diabetes mellitus, debilitating mental diseases, liver or renal failure or structural damage of the gastrointestinal tract. Concomitant medication was taken into consideration. Plasma lipid profiles were quantified using ultra high performance liquid chromatography combined with mass spectroscopy. Subsequently, the identification of lipid molecules was made according to available online databases.

All included patients were diagnosed with either colonic Crohn's disease $(n=5)$ or left sided or extensive ulcerative colitis $(\mathrm{n}=17)$. The control group included 24 subjects. All patients had active disease, confirmed by colonoscopy with targeted biopsy (Table I).

Serum metabolic pathway analysis suggested that the most involved pathways are those of glycerophospholipids, linoleic acid, and sphingolipids, the match status being $2 / 39$ for glycerophospholipid pathways $(\mathrm{p}=0.0007), 1 / 15(\mathrm{p}<0.02)$ and $1 / 25(\mathrm{p}<0.03)$ for linoleic acid and sphingolipid metabolism, respectively. After applying univariate and multivariate analysis, 6 molecules were found to be statistically significant in discriminating patients from healthy controls, while respecting the following: a p-value $<0.05$, a false discovery rate $(\mathrm{FDR})<$ 0.05 and AUROC values higher than 0.8. Thus, tetracosanoic acid, phosphatidylcholine (PC), lysophosphatidylcholine (LPC), sphingomyelin (SM) and diacylglycerol were significantly lower in patients than in healthy controls, while cholesteryl ester (18:3) was significantly higher.

Our results (Fig. 1) show that both PC and LPC have lower values in the IBD group. Sustained inflammation leading to a compromised integrity of the bowel and digestive loss could account for this. However, TNF- $\alpha$ signaling, activation of NF$\kappa \mathrm{B}$, mitogen-activated protein kinase (MAPK) pathway and peroxisome proliferator activated receptor (PPAR) signaling seem to be implicated as well [2]. In a similar fashion to PC, the lower $S M$ values encountered in patients with IBD seem to be a consequence of the compound action of TNF- $\alpha$, NF- $\mathrm{kB}$ and IFN- $\gamma$, which activate sphingomyelinases [3, 4]. Compared to other studies [5], in our study cholesteryl esters showed higher values in patients with IBD than in healthy subjects. These changes could be explained by lipid mobilization or by an increased intestinal permeability.

Our study displayed significant diagnostic power in comparing IBD patients versus control subjects, as the most significant molecules identified with a variable importance in projection (VIP) score $>1.5$ also have ROC curves with values over 0.8 . This is an important clinical finding, as it would be very useful to have serum markers that allow for the diagnosis and monitoring of patients with IBD. Avoiding repeated endoscopies in these patients reduces the costs and risks of the procedures, as well as the psychological impact on patients.

This preliminary study suggests that lipidomic profiling of the serum has the potential to become a diagnostic tool for non-invasive identification of IBD patients.

Cristian Tefas ${ }^{1}$, Carmen Socaciu ${ }^{2}$, Corina Moraru ${ }^{2}$, Marcel Tanțău ${ }^{1}$ 1) Iuliu Hațieganu University of Medicine and Pharmacy, Regional Institute of Gastroenterology and Hepatology, Cluj-Napoca; 2) RTD Center for Applied Biotechnology. BIODIATECH, SC Proplanta, Cluj-Napoca, Romania

Correspondence: Cristian Tefas, tefascristian@gmail.com

Conflicts of interest: None.

DOI: $10.15403 /$ jgld-188

Acknowledgements: The authors would like to thank Horia Ștefănescu for his assistance in writing the manuscript and Andreea Benea for her contribution in preparing the biochemical samples.

\section{REFERENCES}

1. Sewell GW, Hannun YA, Han X, et al. Lipidomic profiling in Crohn's disease: Abnormalities in phosphatidylinositols, with preservation of ceramide, phosphatidylcholine and phosphatidylserine composition. Int J Biochem Cell Biol 2012;44:1839-1846. doi:10.1016/j. biocel.2012.06.016

2. Schneider H, Braun A, Füllekrug J, Stremmel W, Ehehalt R. Lipid based therapy for ulcerative colitis-modulation of intestinal mucus membrane phospholipids as a tool to influence inflammation. Int J Mol Sci 2010;11:4149-4164. doi:10.3390/ijms11104149

3. Kim MY, Linardic C, Obeid L, Hannun Y. Identification of sphingomyelin turnover as an effector mechanism for the action of tumor necrosis factor alpha and gamma-interferon. Specific role in cell differentiation. J Biol Chem 1991;266:484-489.

4. Schütze S, Potthoff K, Machleidt T, Berkovic D, Wiegmann K, Krönke M. TNF activates NF- $\kappa B$ by phosphatidylcholine-specific phospholipase C-induced "Acidic" sphingomyelin breakdown. Cell 1992;71:765-776. doi:10.1016/0092-8674(92)90553-O

5. Fan F, Mundra PA, Fang L, et al. Lipidomic profiling in inflammatory bowel disease: Comparison between ulcerative colitis and Crohn's disease. Inflamm Bowel Dis 2015;21:1511-1518. doi:10.1097/ MIB.0000000000000394 